\title{
Evaluation of the 2006 revision of the medical payment system in Japan by a new estimator of the power transformation model - An analysis of the length of the hospital stay for cataract operations
}

\author{
Nawata, K. ${ }^{\text {a }}$ and K. Kawabuchi ${ }^{\text {b }}$ \\ ${ }^{a}$ Graduate School of Engineering, University of Tokyo, Bunkyo-ku, Tokyo 113-8656, Japan \\ ${ }^{b}$ Section of Health Care Economics, Tokyo Medical and Dental University, Bunkyo-ku, Tokyo, 113-8510, \\ Japan \\ Email:nawata@tmi.t.u-tokyo.ac.jp
}

\begin{abstract}
Since Japanese medical expenses have been increasing rapidly with aging of the population, shortening the average length of stay (ALOS) by reducing long-term hospitalizations has become an important political issue in Japan. A new inclusive payment system based on the Diagnosis Procedure Combination (DPC) was introduced in 82 special functioning hospitals (i.e., university hospitals, the National Cancer Center and the National Cardiovascular Center) in April 2003 in Japan. The DPC Evaluation Division of the Central Social Insurance Medical Council (2010) started to call the new inclusive payment system based on the DPC the DPC/PDPS (per diem payment system), and we use this term and refer to hospitals participating in the DPC/PDPS as DPC hospitals throughout this paper. Since April 2004, the DPC/PDPS has been gradually extended to general hospitals, and it has been revised every two years after that. As of July 2010, a total of 1,391 hospitals, about $18 \%$ of the 7,714 general hospitals in Japan, had joined the DPC system. These DPC hospitals have 458,707 beds, which represents about a half of the total number of beds (909,337 beds) in all general hospitals. The introduction of the DPC/PDPS was one of the largest and most important revisions of the payment system since the Second World War. To ensure the effective use of medical resources, it is absolutely necessary to thoroughly analyze the DPC/PDPS. However, sufficient evaluations of the system have not yet been done. Empirical studies of the LOS and medical payments using econometric models are necessary to evaluate the system correctly. A simple comparison of the ALOS by hospital is not sufficient; differences in types of disease must be considered, and the individual characteristics of patients and types of treatment must also be considered for the same disease.
\end{abstract}

The Box-Cox (1964) transformation model (hereafter, the BC model) is widely used to examine various problems in survival analysis, such as the LOS. However, since the error terms cannot have a normal distribution except in the case where the transformation parameter is zero, the likelihood function under the normality assumption (hereafter, the $\mathrm{BC}$ likelihood function) is misspecified and the maximum likelihood estimator (hereafter, the BC MLE) cannot be consistent. Alternative versions of the BC model have been proposed by various authors. However, because the simplicity of the model is lost with these versions (Showalter, 1994), these alternatives have not been widely used.

In this paper, we first propose a new estimator of the power transformation model (the Box-Cox transformation model excluding the case in which the transformation parameter is zero). The estimator is a modification of the BC MLE and proved to be consistent. We then evaluate the effects of the 2006 Revision of the DPC/PDPS on the LOS and the medical payment for cataract operations (DPC category code: 020110). The number of cataract patients in Japan has been increasing rapidly with the aging of the population. According to a survey conducted by the Ministry of Health, Labour and Welfare (2008), nearly 800,000 cataract operations are performed annually and nearly 2.5 billion yen are spent for cataract operations annually. In the case of cataract operations, a major change was made concerning the DPC classifications, the three periods, and inclusive payments determined by DPC/PDPS in the 2006 Revision. To evaluate the revision, we analyzed the data collected from 20 DPC hospitals before and after the revision. The number of patients in the data set is 4,394 .

Keywords: Diagnosis Procedure Combination (DPC), inclusive payment system, cataract, length of stay (LOS), Box-Cox transformation model, power transformation model 
Nawata and Kawabuchi, Evaluation of the 2006 revision of the medical payment system in Japan by a new estimator of the power transformation model

\section{INTRODUCTION}

Since Japanese medical expenses have been increasing rapidly with aging of the population, shortening the average length of stay (ALOS) by reducing long-term hospitalizations has become an important political issue in Japan. A new inclusive payment system based on the Diagnosis Procedure Combination (DPC) was introduced in 82 special functioning hospitals (i.e., university hospitals, the National Cancer Center and the National Cardiovascular Center) in April 2003 in Japan. The DPC Evaluation Division of the Central Social Insurance Medical Council (2010) started to call the new inclusive payment system based on the DPC the DPC/PDPS (per diem payment system), and we use this term and refer to hospitals participating in the DPC/PDPS as DPC hospitals throughout this paper. Since April 2004, the DPC/PDPS has been gradually extended to general hospitals, and it has been revised every two years after that. As of July 2010, a total of 1,391 hospitals, about $18 \%$ of the 7,714 general hospitals in Japan, had joined the DPC system. These DPC hospitals have 458,707 beds, which represents about a half of the total number of beds $(909,337$ beds) in all general hospitals. The introduction of the DPC/PDPS was one of the largest and most important revisions of the payment system since the Second World War. To ensure the effective use of medical resources, it is absolutely necessary to thoroughly analyze the DPC/PDPS. However, sufficient evaluations of the system have not yet been done. Empirical studies of the LOS and medical payments using econometric models are necessary to evaluate the system correctly. A simple comparison of the ALOS by hospital is not sufficient; differences in types of disease must be considered, and the individual characteristics of patients and types of treatment must also be considered for the same disease.

The Box-Cox (1964) transformation model (hereafter, the BC model) is widely used to examine various problems in survival analysis, such as the LOS. However, since the error terms cannot have a normal distribution except in the case where the transformation parameter is zero, the likelihood function under the normality assumption (hereafter, the $\mathrm{BC}$ likelihood function) is misspecified and the maximum likelihood estimator (hereafter, the BC MLE) cannot be consistent. Alternative versions of the $\mathrm{BC}$ model have been proposed by various authors. However, because the simplicity of the model is lost with these versions (Showalter, 1994), these alternatives have not been widely used.

In this paper, we first propose a new estimator of the power transformation model (the Box-Cox transformation model excluding the case in which the transformation parameter is zero). The estimator is a modification of the BC MLE and proved to be consistent. We then evaluate the effects of the 2006 Revision of the DPC/PDPS on the LOS and the medical payment for cataract operations (DPC category code: 020110). The number of cataract patients in Japan has been increasing rapidly with the aging of the population. According to a survey conducted by the Ministry of Health, Labour and Welfare (2008), nearly 800,000 cataract operations are performed annually and nearly 2.5 billion yen are spent for cataract operations annually. In the case of cataract operations, a major change was made concerning the DPC classifications, the three periods, and inclusive payments determined by DPC/PDPS in the 2006 Revision. To evaluate the revision, we analyzed the data collected from 20 DPC hospitals before and after the revision. The number of patients in the data set is 4,394 .

\section{MODELS}

\subsection{A new consistent estimator for the power transformation model}

We consider the simple power transformation model

$$
z_{t}=x_{t}{ }^{\prime} \beta+u_{t}, \quad z_{t}=y_{t}^{\lambda}, \quad y_{t} \geq 0, t=1,2, \ldots, T,
$$

where $y_{t}$ is the LOS, $x_{t}$ and $\beta$ are k-th dimensional vectors of explanatory variables and the coefficients, respectively, and $\lambda$ is the transformation parameter. Random variables $\left\{u_{t}\right\}$ are independent and identically distributed (i.i.d.) and follow a distribution whereby the support is bounded from below, the first and third moments are zero, and the sixth moment exists and is finite (i.e., $f(u)=0$ if $u \leq-a$ for some $a>0$ where $f(u)$ is the probability density function, $E\left(u_{t}\right)=E\left(u_{t}^{3}\right)=0$, and $\left.E\left(u_{t}^{6}\right)=M_{6}<\infty\right)$. We do not have to assume a specific distribution, and the model is semiparametric in this sense. $\left\{x_{t}\right\}$ are i.i.d. random variables with the finite third moment. $\left\{u_{t}\right\}$ and $\left\{x_{t}\right\}$ are independently distributed. For the identification of the model, the distribution of $x_{t}$ and the parameter space of $\beta$ are restricted so that $\inf \left(x_{t}{ }^{\prime} \beta_{0}\right)-a>0$, where $\beta_{0}$ is the true parameter value of $\beta$ and $\inf \left(x_{t}^{\prime} \beta\right)>c$ for some $c>0$ in the neighborhood of $\beta_{0}$. Unlike the case under the normality assumption, $y_{t}>0$ under this assumption, and we can obtain a consistent model. (Let $\left(y_{t}^{\lambda}-1\right) / \lambda=x_{t}^{*} \beta^{*}+v_{t}$ and $v_{t}=u_{t} / \lambda$, in which case we obtain the BC model. However, to ensure the asymptotic distribution of the estimator, we only considered the $\lambda \neq 0$ case and did not consider the $\lambda=0$ case. Therefore, we call this model a power transformation model rather than a $\mathrm{BC}$ model.) 
Nawata and Kawabuchi, Evaluation of the 2006 revision of the medical payment system in Japan by a new estimator of the power transformation model

Let $\theta^{\prime}=\left(\lambda, \beta^{\prime}, \sigma^{2}\right)$. The BC likelihood function is given by

$$
\log L(\theta)=\sum_{t}\left[\log \phi\left\{\left(z_{t}-x_{t}^{\prime} \beta\right) / \sigma\right\}-\log \sigma\right]+\sum_{t}\left\{\log \lambda+(\lambda-1) \log y_{t}\right\},
$$

where $\phi$ is the probability density function of the standard normal assumption and $\sigma^{2}$ is the variance of $u_{t}$. The BC MLE is obtained as follows:

$$
\begin{aligned}
& \frac{\partial \log L}{\partial \lambda}=-\frac{1}{\sigma^{2}} \sum_{t}\left(z_{t}-x_{t}{ }^{\prime} \beta\right) \log \left(y_{t}\right) z_{t}+\sum_{t} \log \left(y_{t}\right)+n / \lambda=0 \\
& \frac{\partial \log L}{\partial \beta}=\frac{1}{\sigma^{2}} \sum_{t} x_{t}\left(z_{t}-x_{t}{ }^{\prime} \beta\right)=0, \text { and } \frac{\partial \log L}{\partial \sigma^{2}}=\sum_{t} \frac{\left(z_{t}-x_{t}{ }^{\prime} \beta\right)^{2}-\sigma^{2}}{2 \sigma^{4}}=0 .
\end{aligned}
$$

Let $\theta_{0}{ }^{\prime}=\left(\lambda_{0}, \beta_{0}{ }^{\prime}, \sigma_{0}^{2}\right)$ be the true parameter value of $\theta$. Since $E\left[\left.\frac{\partial \log L}{\partial \lambda}\right|_{\theta_{0}}\right] \neq 0$, the BC MLE cannot be consistent. Here, instead of $\partial \log L / \partial \lambda$, we use

$$
G_{T}(\theta)=\frac{1}{\sigma^{2}} \sum_{t}\left(z_{t}-x_{t}{ }^{\prime} \beta\right)\left\{\log \left(x_{t}^{\prime} \beta\right)+\frac{z_{t}-x_{t}{ }^{\prime} \beta}{x_{t}{ }^{\prime} \beta}\right\} z_{t}+\sum_{t}\left\{\log \left(x_{t}{ }^{\prime} \beta\right)+\frac{z_{t}-x_{t}{ }^{\prime} \beta}{x_{t}{ }^{\prime} \beta}\right\}+n \equiv \sum_{t} g_{t}(\theta),
$$

$G_{T}(\theta)$ is obtained by the approximation of $\partial \log L / \partial \lambda$. We consider the roots of the equations, as follows:

$$
G_{T}(\theta)=0, \frac{\partial \log L}{\partial \beta}=0, \text { and } \frac{\partial \log L}{\partial \sigma^{2}}=0 .
$$

Since $E\left[G_{T}\left(\theta_{0}\right)\right]=0$, the estimator obtained by Equation (5) is consistent unlike the BC MLE and we obtain the following proposition:

\section{Proposition 1}

Among the roots of Equation (5), there exists a consistent root.

Let $\hat{\theta}^{\prime}=\left(\hat{\lambda}, \hat{\beta}^{\prime}, \hat{\sigma}^{2}\right)$ be the consistent root. The asymptotic distribution of $\hat{\theta}$ is obtained by the following proposition:

Proposition 2

The asymptotic distribution of $\hat{\theta}$ is given by

$$
\sqrt{T}\left(\hat{\theta}-\theta_{0}\right) \rightarrow N\left[0, A^{-1} B\left(A^{\prime}\right)^{-1}\right],
$$

where $A=-E\left[\left.\frac{\partial \ell_{t}(\theta)}{\partial \theta^{\prime}}\right|_{\theta_{0}}\right]=-\left[\begin{array}{lll}A_{11} & A_{12} & A_{13} \\ A_{21} & A_{22} & A_{23} \\ A_{31} & A_{32} & A_{33}\end{array}\right], A_{11}=E\left[\left.\frac{\partial g_{t}}{\partial \lambda}\right|_{\theta_{0}}\right], A_{12}=E\left[\left.\frac{\partial g_{t}}{\partial \beta^{\prime}}\right|_{\theta_{0}}\right], A_{13}=E\left[\left.\frac{\partial g_{t}}{\partial \sigma^{2}}\right|_{\theta_{0}}\right]$,

$$
\begin{aligned}
& A_{21}=E\left[\left.\frac{\partial \xi_{t}}{\partial \lambda}\right|_{\theta_{0}}\right], A_{22}=E\left[\left.\frac{\partial \xi_{t}}{\partial \beta^{\prime}}\right|_{\theta_{0}}\right], A_{23}=A_{32}{ }^{\prime}=E\left[\left.\frac{\partial \xi_{t}}{\partial \sigma^{2}}\right|_{\theta_{0}}\right]=E\left[\left.\frac{\partial \varsigma_{t}}{\partial \beta}\right|_{\theta_{0}}\right], A_{31}=E\left[\left.\frac{\partial \varsigma_{t}}{\partial \lambda}\right|_{\theta_{0}}\right], \\
& B=E\left[\ell_{t}\left(\theta_{0}\right) \ell_{t}\left(\theta_{o}\right)^{\prime}\right]=\left[\begin{array}{lll}
B_{11} & B_{12} & B_{13} \\
B_{21} & B_{22} & B_{23} \\
B_{31} & B_{32} & B_{33}
\end{array}\right], B_{11}=E\left[g_{t}\left(\theta_{0}\right)^{2}\right], B_{12}{ }^{\prime}=B_{21}=E\left[g_{t}\left(\theta_{0}\right) \xi_{t}\left(\theta_{0}\right)\right], \\
& B_{13}=B_{31}=E\left[g_{t}\left(\theta_{0}\right) \varsigma_{t}\left(\theta_{0}\right)\right] n, B_{22}=E\left[\xi_{t}\left(\theta_{0}\right) \xi_{t}\left(\theta_{0}\right)^{\prime}\right], B_{23}=B_{32}{ }^{\prime}=E\left[\xi_{t}\left(\theta_{0}\right) \varsigma_{t}\left(\theta_{0}\right)\right], B_{33}=E\left[\varsigma_{t}\left(\theta_{0}\right)^{2}\right],
\end{aligned}
$$


Nawata and Kawabuchi, Evaluation of the 2006 revision of the medical payment system in Japan by a new estimator of the power transformation model

$\ell_{t}(\theta)^{\prime}=\left[g_{t}(\theta), \xi_{t}(\theta)^{\prime}, \varsigma_{t}(\theta)\right], \xi_{t}(\theta)=\frac{1}{\sigma^{2}} x_{t}\left(z_{t}-x_{t}^{\prime} \beta\right)$, and $\varsigma_{t}(\theta)=\frac{\left(z_{t}-x_{t}{ }^{\prime} \beta\right)-\sigma^{2}}{2 \sigma^{2}}$.

[Proof]

Let

$$
\ell(\theta)^{\prime}=\sum_{t} \ell_{t}(\theta)^{\prime}=\left[G_{T}(\theta), \frac{\partial \log L}{\partial \beta^{\prime}}, \frac{\partial \log L}{\partial \sigma^{2}}\right]
$$

Then

$$
\sqrt{T}\left(\hat{\theta}-\theta_{0}\right)=-\left[\left.\frac{1}{T} \frac{\partial \ell}{\partial \theta^{\prime}}\right|_{\theta^{*}}\right]^{-1} \frac{1}{\sqrt{T}} \ell\left(\theta_{0}\right),
$$

where $\theta^{*}$ is some value between $\hat{\theta}$ and $\theta_{0}$. Since $E\left[\ell_{t}\left(\theta_{0}\right)\right]=0$ and $\left\{\ell_{t}\left(\theta_{0}\right)\right\}$ are i.i.d. random variables, we get

$$
\frac{1}{\sqrt{T}} \ell\left(\theta_{0}\right) \rightarrow N(0, B)
$$

from the multivariate central limit theorem. Since all elements of $\partial \ell / \partial \theta$ are differentiable,

$$
-\left.\frac{1}{T} \frac{\partial \ell(\theta)}{\partial \theta^{\prime}}\right|_{\theta^{*}} \stackrel{P}{\longrightarrow} A
$$

from Theorem 4.1.4 in Amemiya (1985, pp. 112-113). From Theorem 4.1.3 in Amemiya (1985, pg. 111), the asymptotic distribution of $\hat{\theta}$ is given by (6).

\subsection{A test of the "small $\sigma$ " assumption}

The BC MLE is generally inconsistent. However, if $\sigma_{0} / x_{t}{ }^{\prime} \beta_{0} \rightarrow 0$ and $P\left[y_{t}<0\right] \rightarrow 0$ (in practice, $P\left[y_{t}<0\right]$ is small enough) under the normality for all observations, the BC MLE performs well and we can use it. Following Bickel and Doksum (1981), we call this the "small $\sigma$ " assumption. Under the "small $\sigma$ " assumption the normality assumption is not necessary and we get the "small $\sigma$ asymptotics" of the BC MLE $\hat{\theta}_{B C}^{\prime}=\left(\widehat{\lambda}_{B C}, \widehat{\beta}_{B C}^{\prime}, \hat{\sigma}_{B C}^{2}\right)$ given by

$$
\sqrt{n}\left(\hat{\theta}_{B C}-\theta_{0}\right) \rightarrow N\left(0, C^{-1} B C^{-1}\right)
$$

where $C=-E\left[\left.\frac{\partial^{2} \log L}{\partial \theta \partial \theta^{\prime}}\right|_{\theta_{0}}\right]=-\left[\begin{array}{lll}C_{11} & C_{12} & C_{13} \\ C_{21} & C_{22} & C_{23} \\ C_{31} & C_{23} & C_{33}\end{array}\right] C_{11}=E\left[\left.\frac{\partial^{2} \log L}{\partial \lambda^{2}}\right|_{\theta_{0}}\right], C_{12}{ }^{\prime}=C_{21}=C E\left[\left.\frac{\partial^{2} \log L}{\partial \lambda \partial \beta}\right|_{\theta_{0}}\right]$,

$$
C_{13}=C_{31}=E\left[\left.\frac{\partial^{2} \log L}{\partial \lambda \partial \sigma^{2}}\right|_{\theta_{0}}\right], C_{22}=E\left[\left.\frac{\partial^{2} \log L}{\partial \beta \partial \beta^{\prime}}\right|_{\theta_{0}}\right], C_{23}{ }^{\prime}=C_{32}=E\left[\left.\frac{\partial^{2} \log L}{\partial \beta \partial \sigma^{2}}\right|_{\theta_{0}}\right] \text {, and } C_{33}=E\left[\left.\frac{\partial \log L}{\partial\left(\sigma^{2}\right)^{2}}\right|_{\theta_{0}}\right] \text {. }
$$

Let

$$
A^{*} \equiv A^{-1}, \quad A^{*}=\left[\begin{array}{ccc}
A_{11}^{*} & A_{12}^{*} & A_{13}^{*} \\
A_{21}^{*} & A_{22}^{*} & A_{23}^{*} \\
A_{31}^{*} & A_{32}^{*} & A_{33}^{*}
\end{array}\right], \quad C^{*} \equiv C^{-1}, \quad \text { and } C^{*}=\left[\begin{array}{ccc}
C_{11}^{*} & C_{12}^{*} & C_{13}^{*} \\
C_{21}^{*} & C_{22}^{*} & C_{23}^{*} \\
C_{31}^{*} & C_{32}^{*} & C_{33}^{*}
\end{array}\right]
$$

$A_{i j}^{*}$ and $C_{i j}^{*}$ are submatrices of $A^{*}$ and $C^{*}$ whose locations correspond to $A_{i j}$ and $C_{i j}$, respectively. Let $\hat{\lambda}_{N}$ be the proposed estimator of $\lambda$. Since $G_{T}\left(\theta_{0}\right)=\left.\frac{\partial \log L}{\partial \lambda}\right|_{\theta_{0}}$ under the "small $\sigma$ " assumption, we get

$$
\sqrt{T}\left(\hat{\lambda}_{N}-\hat{\lambda}_{B C}\right) \rightarrow N(0, d)
$$


Nawata and Kawabuchi, Evaluation of the 2006 revision of the medical payment system in Japan by a new estimator of the power transformation model

where $\quad d=p \lim _{n \rightarrow \infty} T \cdot V\left(\hat{\lambda}_{N}-\hat{\lambda}_{B C}\right)=\left(A_{11}^{*}-C_{11}^{*}\right)^{2} B_{11}+\left(A_{12}^{*}-C_{12}^{*}\right) B_{22}\left(A_{12}^{*}-C_{12}^{*}\right)^{\prime}+\left(A_{13}^{*}-C_{13}^{*}\right)^{2} B_{33}$

$$
+2\left(A_{11}^{*}-C_{11}^{*}\right)\left(A_{12}^{*}-C_{12}^{*}\right) B_{12}^{\prime}+2\left(A_{11}^{*}-C_{11}^{*}\right)\left(A_{13}^{*}-C_{13}^{*}\right) B_{13}
$$

Using $t=\sqrt{T}\left(\hat{\lambda}_{N}-\hat{\lambda}_{B C}\right) / \sqrt{\hat{d}}$ as the test statistic, where $\hat{d}$ is the estimator of $d$, we can test the "small $\sigma$ "assumption; that is, we can test whether we can successfully use the BC MLE.

\section{DATA AND THE SUMMARY OF THE 2006 REVISION OF THE DPC/PDPS FOR CATARACT OPERATIONS}

\subsection{Data}

In this study, we use the data of the Section of Health Care Economics of Tokyo Medical and Dental University. The data was collected from 86 hospitals in Japan from 2005 to 2007, during April to December of each year. For each patient, the DPC code, dates of hospitalization and discharge from the hospital, date of birth, sex, placement after hospitalization, ICD-10 code for the principle disease, purpose of hospitalization, presence of concurrent disease and the attending treatment if any, and medical payment amounts (including DPC-based, fee-for-service, and total payments) were reported.

In Japan, in addition to one-eye cataract operations (a single eye is operated on during a single period of hospitalization), two-eye cataract operations (both eyes are operated on during a single period of hospitalization) are also performed. It is natural that the two-eye operation requires a patient to stay in a hospital for a longer period of time. Therefore, we considered patients who underwent one-eye cataract operations only (the DPC code for this procedure after the 2006 revision is $020110 \times x 97 \times 0 \times 0$.) To evaluate the effect of the 2006 Revision of the DPC/PDPS, we used the data set obtained from 20 DPC hospitals (Hp 1-20) where the one-eye cataract operations were performed both before (2005) and after the revision (2006 and 2007, hereafter 2006-7). A total of 4,394 patients were analyzed, 1,078 in 2005 and 3,316 in 2006-7.

In 2005, the ALOS was 4.56 days, the median was 4.0 days, the standard deviation was 2.23 days, the skewness was 3.95, and the kurtosis was 34.87 for all 1,078 patients. The maximum ALOS by hospital was 8.26 days (Hp 19), and the minimum was 2.10 days (Hp 8). The maximum was about four times larger than the minimum, and there were large differences among hospitals. In 2006-7, the ALOS was 4.13 days, the median was 4.0 days, the standard deviation was 1.22 days, the skewness was 1.70 , and the kurtosis was 11.77 for all 3,316 patients. The maximum ALOS by hospital was 6.79 days (Hp 4), and the minimum was 2.40 days ( $\mathrm{Hp} \mathrm{8)}$. The skewness and kurtosis values were large in some hospitals. The large values imply that there were patients who were staying in a hospital for long periods of time.

\subsection{Summary of the revision for cataract operations}

The 2006 Revision of the DPC/PDPS contained a major change for cataract operations. Before the revision, different DPC codes were assigned depending on the presence of concurrent diseases (without concurrent

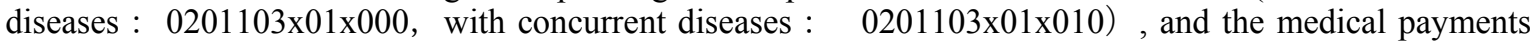
were different accordingly. After the revision, cataract operations were categorized into just one DPC code

$(020110 x x 97 \times 0 x)$ independent of the presence of concurrent diseases. Furthermore, Periods I and II and the Specific Hospitalization Period were shortened, and the per diem inclusive payments were revised as well. The per diem inclusive payment in 2005 for the patients without concurrent diseases was 2,509 points up to the third day of hospitalization, 1,855 points for the 4th-6th days, and 1,577 points for the 7th-10th days. For those with concurrent diseases, the per diem inclusive payment was 2,609 points up to the third day, 2,012 points for the 4th-7th days, and 1,710 points for the 8th-11th days. After the revision, the per diem inclusive payment became 2,418 points up to the second day, 1,787 points for the 3 rd-4th days, and 1,519 points for the 5th-8th days for all cataract patients independent of the presence of concurrent diseases. In 2005, the inclusive payments for 7 days of hospitalization for patient $\mathrm{s}$ without and with concurrent diseases were 14,669 and 15,875 points, respectively. On the other hand, the inclusive payment became 12,967 points after the revision. The inclusive payments were reduced by 1,702 points $(11.6 \%)$ without concurrent diseases and by 2,908 points $(18.3 \%)$ with concurrent diseases.

\section{RESULTS OF THE ESTIMATION}

When we analyze the LOS, it is necessary to consider the characteristics of patients and the types of principal disease as the explanatory variables. For the gender of patients, we use the Female Dummy (1: female, 0: otherwise). The numbers of male and female patients were 1,794 and 2,600, respectively. As a patient becomes older, the LOS tends to increase. Therefore, we used the Age (age of the patient) as an explanatory 
Nawata and Kawabuchi, Evaluation of the 2006 revision of the medical payment system in Japan by a new estimator of the power transformation model

variable. The average and the standard deviation of the age were 73.6 and 10.07 , respectively. To analyze the impact of seasonal climate, we used the Winter Dummy (1: winter, 0: otherwise). The number of patients in winter was 441. The other variables representing characteristics of patients were: Concurrent (number of concurrent diseases), Complication (number of complications), Urgent Dummy (1: urgent hospitalization, 0: otherwise) , and Other Hospital Dummy (1: the patient was discharged to another hospital, 0: otherwise). A total of 418 patients had concurrent diseases. The average number of concurrent diseases for these patients was 1.82. A total of 122 patients had complications, and the average number of complications was 1.18 . The numbers of patients who were urgent hospitalization and were discharged to other hospitals were 45 and 5 , respectively.

Principal Disease Dummies based on the ICD-10 codes were used to analyze the effects of principal diseases. The base of the dummy variables was $\mathrm{H} 25.0$ (senile incipient cataract). The number of patients of $\mathrm{H} 25.0$ was 2,270, H25.1 (senile nuclear cataract) was 200, H25.2 (senile cataract, morgagnian type) was 20, H25.8 (other senile cataract) was 50, $\mathrm{H} 26.0$ (infantile and juvenile cataract) was 35, $\mathrm{H} 26.8$ (other specified cataract) was 5, and H26.9 (unspecified cataract) was 1,814. Twenty Hospital Dummies (1: Hp k, 0: otherwise; ) were used to represent the influences of the hospitals so that did not contain a constant term and contained the same number of dummy variables as the number of hospitals. To analyze the impact of the 2006 Revision of the DPC/PDPS, which is the main purpose of this study, the 2006-7 Dummy (1:2006-7; 0 otherwise) was used. After the revision, the existence of concurrent diseases no longer affected the inclusive payment. To analyze this effect, we added the product of the 2006-7 Dummy and Concurrent to the explanatory variables. Thus $x_{i j}{ }^{\prime} \beta$ of Equation (1) becomes

$$
\begin{aligned}
& x_{t}{ }^{\prime} \beta=\beta_{1} \text { Female Dummy }+\beta_{2} \text { Age }+\beta_{3} \text { Winter Dummy }+\beta_{4} \text { Concurrent }+\beta_{5} \text { Complication } \\
& +\beta_{6} \text { Urgent Dummy }+\beta_{7} \text { Other Hospital Dummy }+\beta_{8} \text { 2006-07 Dummy } \\
& \left.+\beta_{9} \text { (2006-7 Dummy } \times \text { Concurrent }\right)+\sum \beta_{k} k \text {-th Principal Disease Dummy }+\sum \beta_{m} \text { Hpm Dummy. }
\end{aligned}
$$

Table 1 presents the results of the estimation by the newly proposed estimator. For the newly proposed estimator, there are two possible problems: i) Equation (5) has multiple solutions, and ii) Equation (5) does not have a solution. However, just one solution exists in this analysis. The estimate of the transformation parameters is $\hat{\lambda}_{N}=0.2099$, which is significantly smaller than 1.0 ; that implies some patients remained in

the hospital for a long period of time. We also get $\hat{\lambda}_{B C}=0.1893, \hat{d} / \sqrt{T}=0.00270$, and $t=\sqrt{T}\left(\hat{\lambda}_{N}-\hat{\lambda}_{B C}\right) / \hat{d}=7.612$. Therefore, the "small $\sigma$ " assumption is rejected at any reasonable significant level, which means it is not proper to use the BC MLE in this study. The estimates of the Female Dummy and Age are positive and significant at the 5\% and $1 \%$ level, respectively. That implies that the LOS becomes longer if a patient is female and the age becomes higher. The estimate of Concurrent is positive and significant at the 5\% level. That indicates that the concurrent diseases made the LOS longer in 2005, as expected. The estimate of Complication is positive but not significant at the $5 \%$ level and the effect of complications is not admitted. The estimates of Winter, Urgent, and Other Hospital Dummies are not significant at the 5\% level. The estimates of the $\mathrm{H} 25.2$ and $\mathrm{H} 26.0$ Dummies are positive and significant at the $5 \%$ and $1 \%$ levels, respectively. On the other hand, the other types of disease are not significant at the $5 \%$ level. For the estimates of the Hospital Dummies, the maximum is 1.436 (Hp 4), the minimum is 1.151 (Hp 8 ), and the difference between the maximum and minimum values is significantly large compared to other types of variables. There remain large differences among hospitals even if the influence of factors such as patient characteristics and types of principle diseases is eliminated. The estimate of the 2006-7 Dummy is negative and at the $1 \%$ level; that implies the 2006 Revision of the DPC/PDPS reduced the LOS. Moreover, the estimate of the product of the 2006-07 Dummy and Concurrent is negative and at the 5\% level. The coefficient of Concurrent decreased from 0.0117 in 2005 to 0.0001 in 2006-7. This means that the effect of concurrent diseases on the LOS almost disappeared after the revision, and thus the modification concerning the presence of concurrent diseases seems to have had the expected effect.

\section{CONCLUSION}

In this paper, we analyzed the effect of the 2006 Revision of the DPC/PDPS on the LOS and medical payments for single-eye cataract operations (DPC category code 020110) in Japan. We first proposed a new estimator of the power transformation model. Unlike the BC MLE, the proposed estimator is consistent. Using the proposed model, we analyzed the factors that might affect the LOS. We used the data of 4,394 patients collected from 20 DPC hospitals where cataract operations were reported both before and after the revision. We found that the gender, age, and number of concurrent diseases affected the LOS. For principle diseases, we found that $\mathrm{H} 25.2$ and $\mathrm{H} 26.0$ were significant. The ALOSs were significantly different among 
Nawata and Kawabuchi, Evaluation of the 2006 revision of the medical payment system in Japan by a new estimator of the power transformation model

hospitals, despite eliminating the influence of the patient characteristics. The estimates of the 2006-7 Dummy and (2006-07 Dummy $\times$ Concurrent) were negative and significant. After the revision, the effect of concurrent diseases on the LOS almost disappeared, and the modification for the presence of concurrent diseases seems to have had an expected effect. We found that the revision seemed to have a significant impact on the medical payment for the cataract operations. The reduction of medical payment results in a reduction of a hospital's income, and we found that there were large differences in reductions of incomes among hospitals. For some hospitals, the reductions were large amounts, and they might face financial difficulties as a result of the revision. Patients may face serious difficulties if the hospital goes bankrupt and has to stop its operations. Therefore, to improve the DPC/PDPS, we must consider factors such as regional conditions. We also need to perform the same analysis for other diseases. These are subjects for future studies.

\section{REFERENCES}

Amemiya, T . (1985). Advanced Econometrics, Harvard University Press, Cambridge, MA.

Bickel, P. J., and K. A. Doksum. (1981). An Analysis of Transformations Revisited. Journal of the American Statistical Association, 76: 296-311.

Box, G. E. P., and D. R. Cox (1964). An Analysis of Transformation. Journal of the Royal Statistical Society $B, 26: 211-252$.

DPC Evaluation Division, Central Social Insurance Medical Council (2010). "Heisei 24 nendo kaite ni muketa DPC seido (DPC/PDPS) no taiou nit suite (Concerning the Steps for the 2012 Revision of the DPC System (DPC/PDPS))” (in Japanese).

Showalter, M. H. (1994). A Monte Carlo Investigation of the Box-Cox Model and a Nonlinear Least Squares Alternative. Review of Economics and Statistics, 76: 560-570.

Spitzer, J. J. (1984). Variance Estimates in Models with Box-Cox Transformations: Implications for Estimation and Hypothesis Testing. Review of Economics and Statistics, 66: 645-652.

Table 1 Results of estimation

\begin{tabular}{|c|c|c|c|c|c|c|c|}
\hline Variable & Estimate & $\begin{array}{r}\text { Standard } \\
\text { error }\end{array}$ & t-value & Variable & Estimate & $\begin{array}{r}\text { Standard } \\
\text { error }\end{array}$ & t-value \\
\hline Female Dummy & 0.00364 & 0.00159 & 2.2927 & Hp 1 & 1.394 & 0.00791 & 176.1927 \\
\hline Age & 0.0004013 & 0.0000799 & $5.0245^{* *}$ & Hp 2 & 1.3954 & 0.00753 & 185.3183 \\
\hline Winter Dummy & -0.00345 & 0.00263 & -1.3123 & Hp 3 & 1.2743 & 0.01008 & 126.4453 \\
\hline Concurrent & 0.01166 & 0.00529 & $2.2068^{*}$ & Hp 4 & 1.4357 & 0.00881 & 162.9711 \\
\hline Complication & 0.00592 & 0.00409 & 1.4486 & Hp 5 & 1.2396 & 0.00708 & 175.066 \\
\hline Urgent Dummy & 0.00574 & 0.01288 & 0.4457 & Hp 6 & 1.3153 & 0.00647 & 203.3916 \\
\hline $\begin{array}{l}\text { Other Hospital } \\
\text { Dummy }\end{array}$ & -0.01448 & 0.03625 & -0.3993 & Hp 7 & 1.2407 & 0.00742 & 167.2260 \\
\hline \multicolumn{4}{|c|}{ Principal disease dummies } & Hp 8 & 1.151 & 0.00838 & 137.3548 \\
\hline $\mathrm{H} 25.1$ & -0.00312 & 0.0041 & -0.7619 & Hp 9 & 1.3486 & 0.00653 & 206.4274 \\
\hline $\mathrm{H} 25.2$ & 0.02401 & 0.01172 & $2.0483^{*}$ & Нp 10 & 1.3223 & 0.00862 & 153.3689 \\
\hline $\mathrm{H} 25.8$ & 0.0097 & 0.00754 & 1.2867 & Hp 11 & 1.2732 & 0.00844 & 150.8641 \\
\hline $\mathrm{H} 26.0$ & 0.06401 & 0.00985 & $6.4993^{* *}$ & Hp 12 & 1.3037 & 0.00717 & 181.9091 \\
\hline H26.8 & 0.02534 & 0.02324 & 1.0904 & Hp 13 & 1.4231 & 0.00693 & 205.4795 \\
\hline H26.9 & -0.00017 & 0.00299 & -0.0552 & Hp 14 & 1.2486 & 0.00813 & 153.621 \\
\hline \multirow{2}{*}{ 2006-7dummy } & \multirow{2}{*}{-0.01534} & \multirow{2}{*}{0.00198} & \multirow{2}{*}{-7.7602} & Hp 15 & 1.2666 & 0.00674 & 187.9847 \\
\hline & & & & Hp 16 & 1.3194 & 0.00655 & 201.4000 \\
\hline $\begin{array}{l}\text { (2006-7dummy) } \\
\times \text { Concurrent } \\
\end{array}$ & -0.01155 & 0.00548 & $-2.1077^{*}$ & Нp 17 & 1.3847 & 0.00738 & 187.6770 \\
\hline$\lambda$ & 0.2099 & 0.000952 & 220.4802 & Hp 18 & 1.2773 & 0.00839 & 152.2769 \\
\hline \multirow{2}{*}{$R^{2}$} & \multirow{2}{*}{\multicolumn{3}{|c|}{0.603}} & Нр 19 & 1.3609 & 0.00851 & 159.8820 \\
\hline & & & & Hp 20 & 1.3848 & 0.00797 & 173.7903 \\
\hline
\end{tabular}

*:Significant at the 5\% level. **: Significant at the $1 \%$ level. 